\title{
Valores individuales y colectivos en estudiantes universitarios desde la teoría de Schwartz
}

\section{Individual and collective values of university students from the theory of Schwartz}

Claudia María Sánchez H. ${ }^{1}$

\section{Resumen}

El propósito del estudio fue comprender los valores individuales y colectivos de los estudiantes universitarios de la Universidad Pedagógica Nacional Francisco Morazán de Honduras, desde la Teoría de Schwartz, quien propone 10 tipos de valor (poder, logro, hedonismo, autodirección, estimulación, universalismo, benevolencia, tradición, conformidad, seguridad). El Cuestionario de valores de Schwartz (CVS) fue administrado a 321 estudiantes que cursan distintos niveles académicos en sus respectivas carreras de estudio. Los hallazgos indican similitud entre la estructura de valores que la teoría de Schwartz propone y los diez tipos de valor de esta población. Además, se identificó la importancia que otorgan a los valores colectivos en relación a los individuales, lo cual es un aporte en el conocimiento de este constructo.

Palabras clave: valores, estudiantes universitarios, enseñanza superior, universidad

\begin{abstract}
The purpose of the study was to understand the individual and collective values of the university students of the Francisco Morazán National Pedagogical University of Honduras, from Schwartz Theory, who proposes 10 types of value (power, achievement, hedonism, self-direction, stimulation, universalism, benevolence, tradition, compliance, security). The
\end{abstract}

${ }^{1}$ Doctora en Educación. Departamento de Ciencias de la Educación, UPNFM. ORCID 0000-0002-1857-4518. csanchez@upnfm.edu.hn

Recibido 31 de septiembre de 2019 / Aceptado 26 noviembre de 2019 
Schwarz Values Questionnaire (CVS) was administered to 321 students attending different academic levels in their respective study courses. The findings indicate similarity between the structure of values that Schwartz's theory proposes and the ten types of value of this population. In addition, the importance they attach to collective values in relation to individual values was identified, which is a contribution in the knowledge of this construct.

Keywords: values, university students, higher education, university

\section{Introducción}

Las investigaciones sobre jóvenes son relativamente recientes; en la actualidad, se indaga acerca de su perfil socioeconómico, rendimiento académico, participación social, entre otros, y se visualizan como actores importantes en los temas de desarrollo social. Los jóvenes expresan insatisfacción en el diario vivir, plantean muchas preguntas y encuentran pocas respuestas acerca de sus oportunidades y perspectivas de vida. A una buena parte de esta población le ha tocado iniciar su vida laboral, lo que ha implicado dejar sus estudios y los que logran estudiar, lo hacen con notorias limitaciones. El desafío que se presenta para la juventud contemporánea es según Bauman (2005, p. 46) considerar que:

[...] nunca antes estuvimos en una situación semejante. Aún debemos aprender el arte de vivir en un mundo sobresaturado de información. Y también, debemos aprender el aún más difícil arte de preparar a las próximas generaciones para vivir en semejante mundo.

\section{Jóvenes universitarios}

En acuerdo con Martínez, Silva y Hernández (2010, p. 26), “los jóvenes son agentes clave en el proceso de renovación de la sociedad, pues constituyen la generación que se hará cargo de la conducción del país". Los universitarios juegan un papel protagónico y relevante durante sus años de formación profesional, pues son el relevo generacional que la sociedad está requiriendo. El rasgo característico de la situación del estudiante universitario es su transitoriedad; en otras palabras, no se es estudiante toda la vida. Es un periodo provisional en 
el que se generan aspiraciones y proyectos y para quienes se dedican de lleno a la Universidad, implica además del estudio, la convivencia entre iguales, no tener compromisos, estar fuera de la casa y en por lo menos, más de la mitad de los casos, fuera del rigor del trabajo.

Los estudiantes universitarios conforman un grupo poblacional favorecido, por el acceso que tienen de cursar una carrera universitaria, contar con la posibilidad de profundizar en áreas del saber y una vez finalizado sus estudios, lograr una titulación que les habilite para desempeñarse como profesionales en una disciplina específica. Esta condición de los estudiantes universitarios de ser visualizados como un grupo favorecido o privilegiado, genera desde la sociedad cierta expectativa y presión de lo que se debe ser y hacer.

El ingreso a la universidad por parte de los jóvenes va en aumento y en la actualidad se observa una masificación en la educación terciaria. El acceso a la universidad ya no es exclusivo de las élites dominantes, sino que se abre a las clases menos aventajadas que hacen esfuerzos por lograr el ingreso de sus hijos a dichos establecimientos de educación superior y es que “en América Latina en general [... ] la masificación de la universidad parece responder a una dinámica propia, determinada por la evolución económica y social particular de los países de la región" (Gallo, 2005, p. 50).

Pese a las diversas adversidades que enfrentan los estudiantes universitarios en su paso, tanto por ingresar a la universidad como para permanecer en ella, (débil base de estudios de nivel de diversificado, limitaciones económicas, situaciones de equidad social, entre otras), existe una reconocida determinación por contar con estudios de nivel superior y es que "los universitarios, [...], vistos como grupo social de referencia, basan su prestigio social en el atributo de tener una escolaridad superior a la media nacional, aunque su situación socioeconómica y cultural sea sumamente heterogénea" (Suarez y Pérez, 2008, p. 22).

Por ello, los objetivos del estudio pretendieron caracterizar los tipos de valores de los estudiantes, e identificar los valores individuales y colectivos que posee la población estudiantil de la UPNFM, con base en la Teoría de Valores de Schwartz. 


\section{Métodos y materiales}

\section{Diseño}

El estudio de los valores humanos como objeto de estudio, fue abordado desde la aproximación cuantitativa. Para Sampieri, Fernández- Collado y Baptistta (2006, p. 6) “...se pretende explicar y predecir los fenómenos investigados, buscando regularidades y relaciones causales entre elementos. Esto significa que la meta principal es la construcción y demostración de teorías (que explican y predicen)."

La investigación cuantitativa tiene "su soporte en la indagación a través de elementos cognitivos y en datos numéricos extraídos de la realidad, procesados estadísticamente para probar teorías" (Del Canto y Silva, 2013, p. 26).

\section{Población y muestra}

La población estudiada corresponde a jóvenes estudiantes de la Universidad Pedagógica Nacional Francisco Morazán (UPNFM), matriculados en el sistema presencial, sede Tegucigalpa, del II período del año 2016.

La muestra estuvo conformada por 321 estudiantes universitarios, cuya edad oscila entre 17 a 30 años, de los cuales 202 son mujeres y 119 son varones. Los estudiantes pertenecían a las dos facultades existentes en la UPNFM; Facultad de Humanidades (FAHU) y Facultad de Ciencia y Tecnología (FACYT). Se incluyó en la investigación estudiantes universitarios de distintos niveles de avance académico, según el espacio de formación pedagógica (asignaturas/ cursos de formación) que cursaban al momento de la investigación.

\section{Variables}

Las variables de la investigación son las siguientes:

a. Variables socio-demográficas: (sexo, edad, carrera, religión, partido político, año de ingreso, ocupación entre otras).

b. Valores humanos: de los cuales se identifican diez (10) tipos de valores y cuatro (4) 
dimensiones (Schwartz, 1992). Los diez tipos de valores son: 1) poder, 2) logro, 3) hedonismo, 4) estimulación, 5) autodirección, 6) universalismo, 7) benevolencia, 8) tradición, 9) conformidad, y 10) seguridad. Los tipos de valores se agrupan en cuatro dimensiones contrapuestas: a) apertura al cambio versus b) conservación y, d) auto-trascendencia versus e) auto-promoción.

Para los propósitos de este estudio, se adopta la definición de Schwartz y Bilsky (1990, p. 551), para quienes los valores son; “(a) conceptos o creencias, (b) sobre estados finales o comportamientos deseables, (c) que trascienden situaciones específicas, (d) selección de guías o evaluación de comportamientos y eventos, y (e) son ordenados por importancia relativa". Esta definición operativa universal acerca de los valores, integra las cinco características referidas y están contenidas en las definiciones de autores que han abordado este objeto de estudio y que permiten una mejor comprensión del ser humano.

Los 10 tipos de valores que refiere la teoría, se detallan en 57 indicadores que registra el instrumento y se definió el nivel de datos métrico. La tabla 1, detalla los indicadores.

Tabla 1

Indicadores para cada variable del instrumento

\begin{tabular}{ll}
\hline \multicolumn{1}{c}{ Valores (10) } & \multicolumn{1}{c}{ Indicadores (57) } \\
\hline 1. Poder & 3. Poder social \\
& 12. Riqueza \\
& 27. Autoridad \\
2. Hedonismo & 4. Cuido mi imagen pública \\
& 50. Placer \\
3. Conformidad & 57. Indulgente conmigo mismo \\
& 11. Buenos modales \\
& 20. Autodisciplina \\
& 40. Honra a los padre y mayores \\
4. Tradición & 18. Obediente \\
& 32. Mespetar la tradición \\
& 36. Humilde \\
& 44. Acepto lo que me toca en la vida \\
& 51. Devoto \\
\hline
\end{tabular}

2 "values are (a) concepts or beliefs, (b) about a desirable end states or behaviors, (c) that transcend specific situations, (d) guide selection or evaluation of behavior and events, and (e) are ordered by relative importance" (Schwarz \& Bilsky, 1990, p. 551) 
Tabla 1 (continuación)

Indicadores para cada variable del instrumento

\begin{tabular}{|c|c|}
\hline Valores (10) & Indicadores (57) \\
\hline 5. Benevolencia & $\begin{array}{ll}\text { 33. } & \text { Leal } \\
\text { 45. } & \text { Honesto } \\
\text { 49. } & \text { Ayuda } \\
\text { 52. } & \text { Responsable } \\
\text { 54. } & \text { No rencoroso }\end{array}$ \\
\hline 6. Universalismo & $\begin{array}{l}\text { 1. Igualdad } \\
\text { 17. Un mundo en paz } \\
\text { 24. Unión con la naturaleza } \\
\text { 26. Sabiduría } \\
\text { 29. Un mundo de belleza } \\
\text { 30. Justicia social } \\
\text { 35. Tolerante } \\
\text { 38. Protección del medio ambiente }\end{array}$ \\
\hline 7. Autodirección & $\begin{array}{ll}\text { 5. } & \text { Libertad } \\
\text { 16. } & \text { Creatividad } \\
\text { 31. } & \text { Independiente } \\
\text { 41. } & \text { Elijo mis propias metas } \\
\text { 53. } & \text { Curioso }\end{array}$ \\
\hline 8. Estimulación & $\begin{array}{l}\text { 9. Una vida excitante } \\
\text { 25. Una vida variada } \\
\text { 37. Atrevido }\end{array}$ \\
\hline 9. Logro & $\begin{array}{l}\text { 34. Ambicioso } \\
\text { 39. Influyente } \\
\text { 43. Capaz } \\
\text { 55. Triunfador }\end{array}$ \\
\hline 10. Seguridad & $\begin{array}{ll}\text { 8. } & \text { Orden social } \\
\text { 13. } & \text { Seguridad Nacional } \\
\text { 15. } & \text { Reciprocidad de favores } \\
\text { 22. } & \text { Seguridad familiar } \\
\text { 56. } & \text { Limpio }\end{array}$ \\
\hline
\end{tabular}

Nota: Adaptado al español del documento "Keying of SVS Ten Individual Level Value Scales", facilitado por el Dr. Shalom Schwartz. Los 10 tipos de valor se componen de 57 indicadores que permiten su medición.

\section{Instrumentos}

Dos instrumentos fueron empleados en este estudio; a) Cuestionario de valores de Schwartz (CVS) y b) Encuesta Sociodemográfica, la cual incluyó aspectos como edad, sexo, escolaridad de los padres y sí mismo, actividad cotidiana a la que se dedica además de estudiar, carrera que estudia, religión, partido político, año de ingreso, procedencia, entre otras. Ambos 
instrumentos se organizaron en un cuestionario que los participantes del estudio podrían contestar en línea. A continuación, se detalla cada uno de estos instrumentos. Vale decir que el CVS siguió un proceso de validación mediante jueces expertos para su aplicación en el contexto de la UPNFM.

\section{Cuestionario de valores de Schwartz (CVS)}

Para los propósitos de esta investigación se decidió emplear el Cuestionario de Valores de Schwartz (CVS) en su versión inicial. La investigadora tuvo la oportunidad de intercambiar comunicación electrónica en más de una ocasión con el Dr. Shalom Schwartz, autor del instrumento, quien facilitó la versión en español, la cual fue objeto de validación por jueces expertos. Esta versión inicial en nada desmerece las versiones refinadas que recientemente ha trabajado el autor. La versión inicial permite establecer comparaciones que pueden ser similares con las versiones refinadas.

El cuestionario de valores de Schwartz cuenta con 57 ítems, presentados en dos listas de 30 y 27 valores respectivamente. A cada uno de los ítems, se le asocia una escala asimétrica de -1 (menos uno) a 7 (siete), en la que el sujeto debe señalar la importancia de dicho valor como principio- guía en su vida. Los ítems del cuestionario, teóricamente se distribuyen en diez dimensiones supuestamente universales que responden a distintas motivaciones subyacentes a los valores que las integran. Estas dimensiones o tipos motivacionales son hedonismo, estimulación, autodirección, universalismo, benevolencia, conformidad, tradición, seguridad, poder y logro. ${ }^{3}$

\section{Validez y confiabilidad del instrumento}

Con la finalidad de asegurar la validez y confiabilidad del instrumento, en el contexto hondureño, se procedió a efectuar el análisis factorial para determinar la validez de constructo, entendida como la obtención de evidencias que apoyan que las conductas observadas en un test son indicadores del constructo (Tovar, 2007). Respecto al estudio de la confiabilidad se obtuvo el estadístico Alpha de Cronbach que determina la consistencia interna de los ítems.

3 Para mayor detalle del CVS, favor contactar al Dr. Shalom Schwartz al correo electrónico: msshasch@mscc.huji.ac.il 
Los resultados de ambos procedimientos se detallan a continuación:

Validez por jueces expertos

Vale decir que en el proceso de validación del instrumento, a través de jueces expertos, se identificaron especialistas en áreas afines a la Psicología, a los cuales se solicitó examinar el cuestionario y registrar sus opiniones en una tabla que incluía cinco criterios: a) claridad en la redacción, b) coherencia interna, c) introducción de la respuesta (sesgo), d) lenguaje adecuado al nivel del informante y, e) si el instrumento, mide lo que pretende medir, además de una casilla de observaciones por cada ítem. En general, los jueces reportaron claridad del instrumento, señalaron recomendaciones para la mejor comprensión de las instrucciones, mismas que se atendieron en un nuevo documento, el cual obtuvo el visto bueno del Dr. Shalom Schwartz, autor y propietario de los derechos del instrumento.

Validez de constructo

La validez de contenido se analizó por medio del procedimiento de componentes principales. Los resultados obtenidos en las pruebas de adecuación muestral $(\mathrm{KMO}=.90)$ y esfericidad de Barllet $\chi 2=(159)=7922 ; p=.000$, resultaron adecuadas para proceder con el análisis. El análisis de componentes principales reveló 10 factores, o componentes con auto valores superiores a 1 y que coinciden con la estructura del instrumento. La solución de los 10 factores explicó el $57.55 \%$ de la varianza.

\section{Confiabilidad}

La puntuación obtenida es de $(\alpha=.94)$, que indica un alto nivel de fiabilidad del instrumento.

\section{Encuesta sociodemográfica}

Con el objeto de recabar información sobre los participantes se aplicó una encuesta que solicitaba información acerca del sexo, edad, ocupación, estado civil, año de ingreso a la universidad, afiliación religiosa, étnica y política, así como área de procedencia, entre otros. Lo anterior con la intención de describir de mejor manera a los estudiantes de este estudio y realizar los análisis respectivos. 
Se realizó un proceso de validación de ambos instrumentos (integrados en un cuestionario en línea) a 21 estudiantes universitarios, matriculados en el II período académico del año 2016 que poseían las mismas características de los criterios definidos por los participantes, a fin de verificar el nivel de comprensión del cuestionario, los tiempos de respuesta y la claridad de la información.

\section{Discusión teórica}

\section{Teoría de valores de Schwartz}

Este estudio tuvo como base teórica la propuesta de Shalom Schwartz profesor de la Universidad Hebrea de Jerusalén, en la cual da cuenta de la existencia de una estructura universal de los valores humanos.

De acuerdo con Schwartz y Bilsky (1990), los valores son definidos como: (a) conceptos o creencias, (b) sobre estados finales o comportamientos deseables, (c) que trascienden situaciones específicas, (d) selección de guías o evaluación de comportamientos y eventos y (e) son ordenados por importancia relativa. Esta definición operativa universal acerca de los valores, integra las cinco características referidas y están contenidas en las definiciones de autores que han abordado este objeto de estudio y que permiten una mejor comprensión del ser humano.

Brinkmann y Bizama (2000, p. 4) al referirse a la capacidad de los seres humanos de habitar en comunidad y establecer relaciones en el marco de los valores, sostienen que:

[...] para ser miembros efectivos de los grupos, los seres humanos deben comunicarse. Gracias al desarrollo cognitivo, los individuos llegan a ser capaces de representarse las necesidades conscientemente al modo de metas o valores. Los conceptos valóricos culturalmente compartidos se transmiten a los miembros de una cultura a través del proceso de socialización. La adecuada integración de ellos les permitirá la comunicación con los demás en torno a estas metas o valores. 
Este hecho, de que los valores puedan desarrollarse en el marco de una comunidad, ofrece una potente posibilidad de alcanzar propósitos; entre ellos, la adecuada comunicación y el fortalecimiento de una cultura en la que permean valores compartidos que promueven ciudadanía. Según Schwartz (2006, p. 2), “los valores son usados para caracterizar sociedades e individuos, para identificar el cambio a través del tiempo, y para explicar las bases motivacionales de actitudes y conducta". ${ }^{4}$ Schwartz propone una teoría de valores que es consistente en diferentes culturas. Para este autor:

La teoría se refiere a los valores básicos que las personas en todas las culturas reconocen. Identifica las orientaciones de valores motivacionalmente distintas y específica la dinámica del conflicto y la congruencia entre estos valores. Algunos valores se contradicen entre sí (por ejemplo, benevolencia y poder), mientras que otros son compatibles (por ejemplo, conformidad y seguridad). [...]. Si las estructuras de valores son similares a través de grupos culturalmente diversos, esto sugeriría que existe una organización universal de motivaciones humanas. Por supuesto, incluso si los tipos de motivación humana que expresan los valores y la estructura de las relaciones entre ellos son universales, los individuos y los grupos difieren substancialmente en la importancia relativa que atribuyen a sus valores. Es decir, los individuos y los grupos tienen diferentes 'prioridades' de valor o 'jerarquías' (Schwartz, 2006, p. 2).

La propuesta teórica de Schwartz ha sido validada en varias culturas alrededor del mundo, mostrando consistencia en la estructura que se declara. El hecho de que se hable de aspectos motivacionales en los seres humanos permite profundizar en este objeto del conocimiento y entender lo que para las personas es de prioridad o no. Siguiendo con el pensamiento de Schwartz, el considera que:

Cuando pensamos en nuestros valores, pensamos en lo que es importante para nosotros en la vida. Cada uno de nosotros posee numerosos valores (por ejemplo, logro, seguridad,

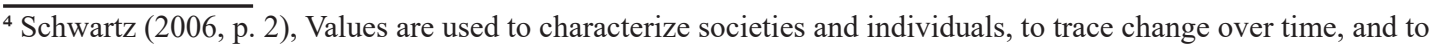
explain the motivational bases of attitudes and behavior (Traducción).
} 
benevolencia) con diversos grados de importancia. Un valor particular puede ser muy importante para una persona, pero poco importante para otra (Schwartz, 2006, pp. 249-250).

Castro y Nader (2006, p. 160) refieren que en:

[...] un estudio desarrollado en más de 40 países, Schwartz encontró que había diez tipos de valores que subyacían a todas las culturas estudiadas. Todos ellos se agrupan en dos bipolaridades (dimensiones) que contrastan, por un lado, la trascendencia de los intereses personales en función de los colectivos (Autotrascendencia) y, en el otro extremo, aquellas personas que priorizan sus intereses propios (Autopromoción), mientras que, por otro lado, algunas personas aprecian la seguridad y el orden (Conservación) y contrariamente, otros valoran la independencia de acciones y pensamientos (Apertura al cambio). A su vez, todas estas polaridades definen intereses.

Los diez valores motivacionales que propone Schwartz, son los siguientes: (1) Poder, (2) Logro, (3) Hedonismo, (4) Estimulación (5) Autodirección, (6) Universalismo, (7) Benevolencia, (8) Tradición, (9) Conformidad, y (10) Seguridad. Al identificar la estructura de valores que se pueden extender universalmente, Schwartz plantea la existencia de diez valores agrupados en dos bipolaridades (dimensiones); por un lado, la trascendencia de los intereses personales frente a los de los colectivos. En la otra dimensión, se ubican las personas que aprecian la seguridad y el orden y contrariamente otras valoran la independencia de acciones y pensamientos (Castro Solano y Nader, 2006, p. 160). "La tipología hedonismo, no se incluye en ningún valor de orden superior ya que podría estar contenida tanto en auto engrandecimiento como en apertura al cambio" (Arciniega y González, 2002, p. 45). La siguiente figura muestra esta representación, en la que se incluye los diez tipos de valor y las cuatro dimensiones que se contraponen entre sí. 


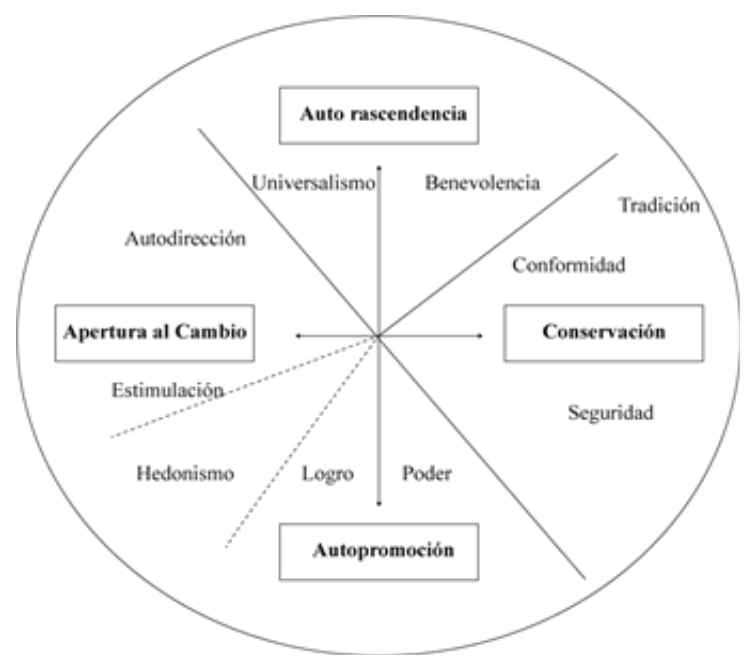

Figura 1. Representación diagramática de la teoría de Schwartz. Adaptado de Astill, Feather y Keeves (2002, p. 347). Se observan las cuatro dimensiones y tipos de valor propuestos por Schwartz, que indica que los valores y dimensiones se contraponen entre sí de acuerdo a la importancia que los individuos otorgan.

\section{Tipos y dimensiones de valores.}

Como ha sido señalado previamente, Schwartz establece diez tipos de valores que han sido constantes en estudios en diferentes países. La siguiente tabla muestra dichos tipos de valor y la motivación a la cual responden.

\section{Tabla 2}

Tipos de valor postulados por Schwartz

N. Tipos de Valores Motivación a la cual responden

1. Poder

2. Logro

3. Hedonismo

4. Estimulación

5. Autodirección

6. Universalismo

7. Benevolencia

8. Tradición

9. Conformidad

10. Seguridad
Dominio de las otras personas y de los recursos.

Éxito social mediante la demostración de competencias.

Placer y gratificación sensorial.

Variedad, novedad y reto en la vida.

Pensamiento independiente y elección de la propia acción.

Aprecio, tolerancia y protección del bienestar de todas las personas y la naturaleza.

Fomento del bienestar de las personas cercanas.

Respeto, aceptación y reproducción de costumbres e ideas tradicionales.

Restricción de impulsos que puedan dañar a otros o violar las expectativas sociales. Seguridad y estabilidad del orden social, de las relaciones y del propio organismo.

Nota: Tomado de Linares, Molpeceres y Musitu (2001, p. 192). Cada tipo de valor, responde a una motivación. 
De acuerdo con Schwartz y Blinsky (1987, p. 551):

[[...] dado que los valores son objetivos, deben representar los intereses de una persona o grupo. [Existen] valores que sirven a los intereses de los individuos (por ejemplo, ambición, placer) versus los intereses colectivistas (por ejemplo, responsabilidad, amabilidad). [...] Algunos valores pueden servir a intereses tanto individualistas como colectivistas (por ejemplo, amor madurez, sabiduría).

En ese sentido, y de acuerdo a Brinkmann y Bizama (2000, p. 7), los "valores que sirven a intereses individualistas son opuestos a los valores que sirven a intereses colectivistas". Schwartz postula que los cinco tipos de valores que sirven primariamente intereses individualistas (poder, logro, hedonismo, estimulo, autodirección) se ubican en forma adyacente, constituyendo una región opuesta a otra que resulta al agrupar los tres tipos de valores que sirven primariamente intereses colectivos (benevolencia, tradición, conformidad). [...] Los tipos de valores universalismo y seguridad, sirven a ambos tipos de fines y, por lo tanto, se ubican en los límites entre estas regiones".

\section{Resultados}

Tipos de valor en estudiantes universitarios

Fue de interés conocer cómo se manifestaban estos diez tipos de valor en los estudiantes universitarios, para obtener mayor comprensión de hacia dónde orientan sus preferencias y a qué otorgan mayor importancia en la vida. La figura siguiente muestra cómo se manifiestan los 10 tipos de valor en los estudiantes universitarios. 


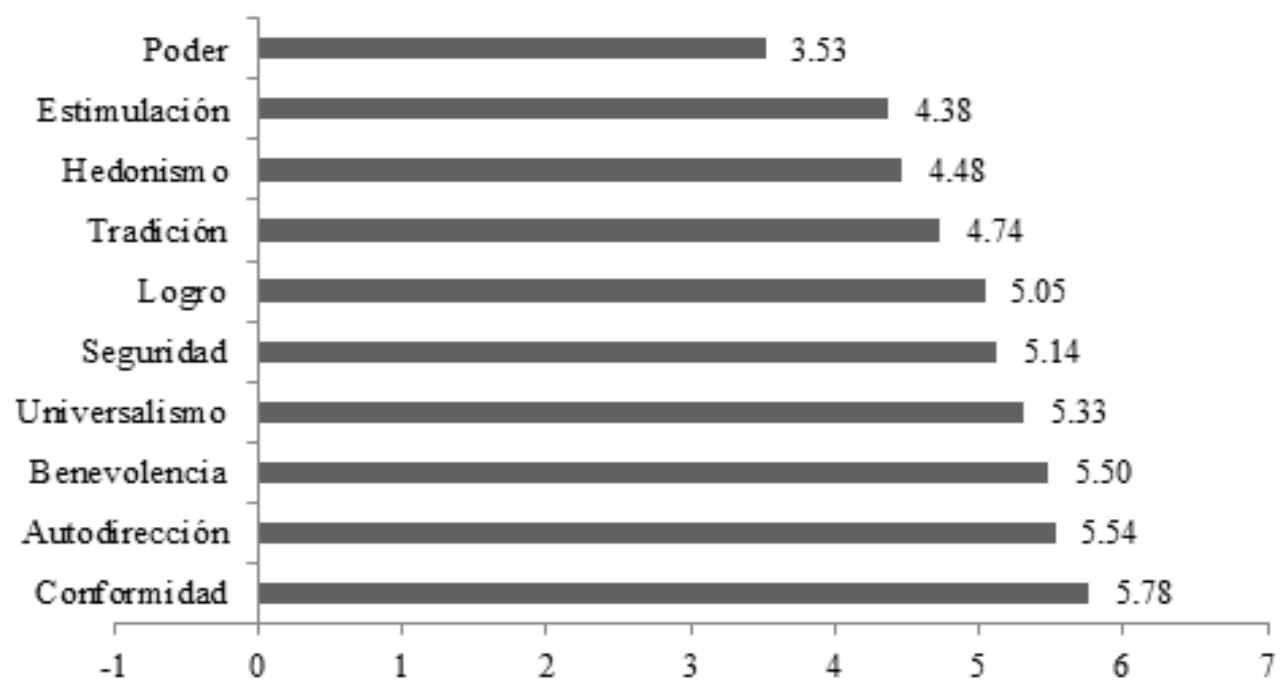

Figura 2. Tipos de valor en estudiantes de la UPNFM. Fuente: Elaboración propia. Se observa los puntajes en orden creciente, de acuerdo a la importancia relativa de los estudiantes universitarios.

En la siguiente tabla, se aprecia los tipos de valor organizados por puntaje de mayor a menor. Es así que conformidad se ubica en el primer lugar (5.78), seguido de autodirección (5.54), y en tercer lugar benevolencia (5.50). El valor poder, resultó el de menor puntaje (3.53), le antecede estimulación (4.38) y hedonismo (4.48).

Tabla 3

Tipos de valor de estudiantes universitarios en orden jerárquico

\begin{tabular}{rlc}
\hline N. & \multicolumn{1}{c}{ Tipo de valor } & Puntaje \\
\hline 1. & Conformidad & 5.78 \\
2. & Autodirección & 5.54 \\
3. & Benevolencia & 5.50 \\
4. & Universalismo & 5.33 \\
5. & Seguridad & 5.14 \\
6. & Logro & 5.05 \\
7. & Tradición & 4.74 \\
8. & Hedonismo & 4.48 \\
9. & Estimulación & 4.38 \\
10. & Poder & 3.53 \\
\hline
\end{tabular}

Nota: Los diez tipos de valor se muestran en orden de mayor a menor puntaje. Fuente: Elaboración propia. 
Los tres tipos de valor que aparecen con mayor puntuación son conformidad, autodirección y benevolencia, con puntajes que van desde 5.78 a 5.50. Por conformidad se entiende, la capacidad de restringir impulsos para evitar daño a otros o quebrantar expectativas sociales. En cuanto a autodirección, esta se refiere a contar con un pensamiento autónomo y realizar propias elecciones. Y benevolencia, que tiene que ver con fomentar el bienestar de personas cercanas.

Es interesante que los puntajes más altos correspondan a tipos de valor que se orientan a intereses distintos, por ejemplo, conformidad tiene que ver con mantener las expectativas sociales y evitar dañar a otros. En tanto que autodirección va orientada a procurar el pensamiento autónomo y tomar propias elecciones. Este resultado es similar a estudios como el llevado a cabo por Veliz, Dorner, Gonzáles y Ripoll (2017) con estudiantes de carreras de la salud. Los tipos de valor conformidad y autodirección obtuvieron mayor puntaje en relación a los demás tipos de valor. De igual manera, en el estudio llevado a cabo por Fasce, Pérez, Ortíz \& Parra (2013), con una muestra de estudiantes universitarios de medicina, se obtuvo resultados de mayor puntaje en estos dos tipos de valor.

De manera que lejos de pensar que son valores que se oponen, es posible pensar que es al interior del individuo mismo, donde se establece esta búsqueda de equilibrar estos principios guías para la vida que promuevan lo personal y lo social. Y en el caso de los estudiantes universitarios, se encuentran en un momento en el que buscan su propia autonomía, pero aún se orientan por restricciones que norman sus vidas.

En contraste, los tipos de valor que aparecen en menor proporción son hedonismo, estimulación y poder con puntajes que van desde 4.48 a 3.53. El hedonismo está referido a buscar el placer y la gratificación personal. En lo referido a la estimulación, tiene que ver con la variedad, lo novedoso y los retos en la vida. Y el poder, tiene que ver con el dominio de las otras personas y de los recursos. Es posible que, en los participantes del estudio, que han decidido por una carrera humanista como es la educación, no prevalezcan aquellos tipos de valor que van encaminados a su engrandecimiento o satisfacción. 
Asimismo, en esta población de estudiantes universitarios, este perfil de tipos de valor indica un predominio de valores colectivos, con un mayor interés en guardar las normas y procurar el interés de los demás y en ese sentido menos centrado en aquellos que favorecen lo personal, como el placer, lo novedoso y el ejercer dominio sobre otros y los bienes materiales.

\section{Valores individuales y colectivos en estudiantes de la UPNFM}

A partir de la Teoría Transcultural de Valores de Schwartz (1992), los valores se articulan en torno a un objetivo instrumental y terminal que expresan intereses individuales, colectivos o ambos, pertenecientes a un dominio motivacional y es evaluado en un rango de importancia como principio rector de vida.

El gráfico siguiente establece los valores individuales en los participantes del estudio.

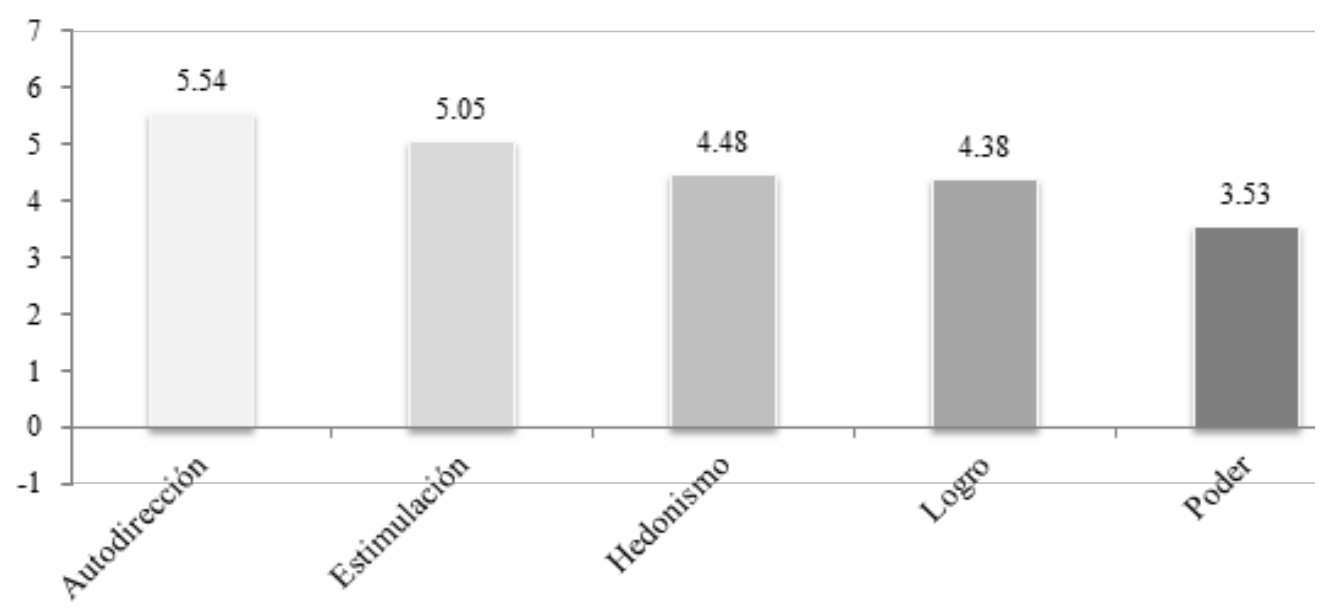

Figura 3. Valores individuales de estudiantes de la UPNFM. Fuente: Elaboración propia. Se observa que, de los cinco valores individuales, autodirección y estimulación son los de mayor puntaje.

La figura muestra que el tipo de valor con mayor puntaje es el de Autodirección (5.54), referido al pensamiento independiente y elección de la propia acción, el segundo valor resultó el de Estimulación (5.05), concerniente a la variedad, novedad y reto en la vida, en tercer lugar, resultó el tipo de valor de Hedonismo con un puntaje de 4.48, que implica búsqueda de placer y gratificación sensorial. Logro resultó con un 4.38, implica éxito social mediante 
la demostración de competencias y Poder con un 3.53, que implica el dominio de las otras personas y de los recursos.

Se observa que, en los valores individuales de hombres y mujeres, Autodirección resultó ser el de mayor puntaje en ambos géneros (en hombres 5.63 y para mujeres 5.60), en segundo lugar, está el tipo de valor Logro, con igual puntaje para ambos géneros (5.05). En tercer lugar, se encuentra Hedonismo (4.57 para hombres y 4.42 para mujeres). En cuarto lugar, Estimulación resultó en segundo lugar, menor en hombres que en mujeres (4.36 y 4.39), finalmente el tipo de valor Poder, resultó en ambos géneros como un tipo de valor de menor puntaje (3.65 en hombres y 3.46 en mujeres).

En cuanto a los valores colectivos, la siguiente gráfica muestra cómo se manifiestan estos valores en los estudiantes universitarios.

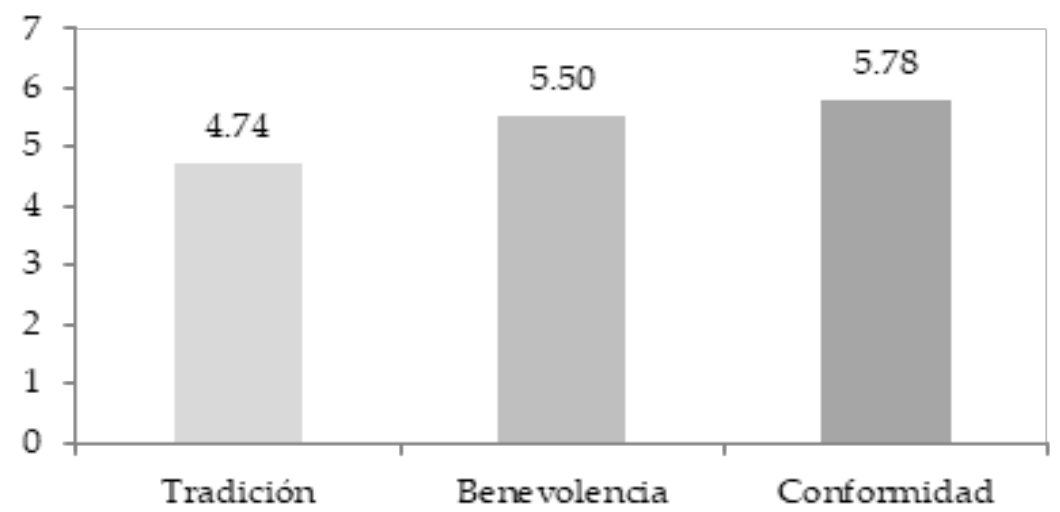

Figura 4. Valores colectivos en estudiantes de la UPNFM. Fuente: Elaboración propia. Se aprecia en la figura que el tipo de valor colectivo de mayor puntaje es conformidad, seguido de benevolencia.

En el caso de los valores colectivos, los estudiantes universitarios muestran una mayor inclinación al valor Conformidad (5.78), en segundo lugar, se ubica la Benevolencia (5.50) y finalmente la Tradición (4.74). Es decir que los jóvenes universitarios dan mayor valor a la restricción de impulsos que puedan dañar a otros o violar las expectativas sociales, además al fomento del bienestar de las personas cercanas y finalmente al respeto, aceptación y reproducción de costumbres e ideas tradicionales. 
Se consideró oportuno comparar el peso de los valores individuales versus los valores colectivos en la población participante de esta investigación, en ese sentido se observa que existe una mayor inclinación a preferir los valores colectivos (5.34) como metas que guían su vida que los valores individuales (4.60). La figura siguiente muestra esta situación

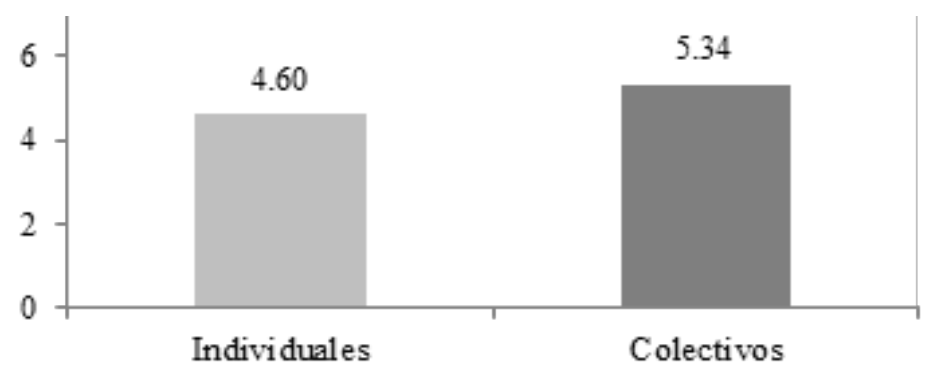

Figura 5. Peso de valores individuales y colectivos. Fuente: Elaboración propia. Al comparar ambos valores se observa mayor puntaje a los valores colectivos.

Lo anterior es congruente con el tipo de universidad en la que los participantes del estudio se forman, pues su principal enfoque es formar profesores, por tanto, los tipos de valor que se toman como principios que guían la vida, tienen que ver con aquellos que se centran en el cuidado y bienestar de los demás antes que el bienestar propio.

Se realizó una comparación entre los valores individuales y colectivos por género. El siguiente gráfico muestra estos resultados.

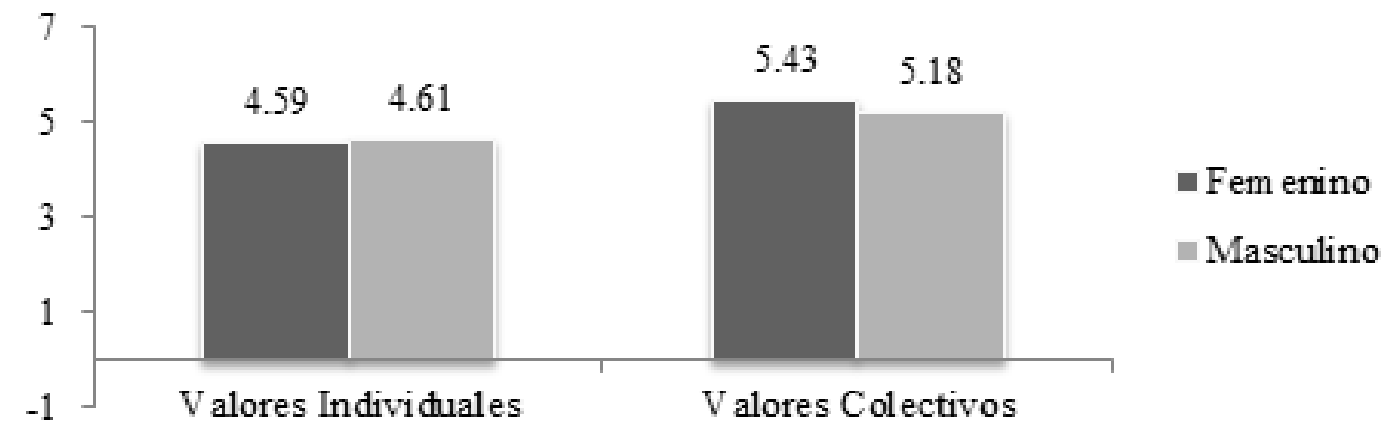

Figura 6. Valores individuales y colectivos por género. Fuente: Elaboración propia. Un mayor puntaje de las mujeres respecto de los hombres en los valores colectivos. En los valores individuales los puntajes son bastante cercanos. 
En relación a cómo se manifiestan estos valores individuales y colectivos en hombres y mujeres, se aprecia que los hombres puntuaron levemente más alto (4.61) que las mujeres (4.59) en los valores individuales. Y las mujeres puntuaron más alto en los valores colectivos (5.43) en relación a los hombres (5.18)

\section{Dimensiones de los tipos de valor}

Al realizar el análisis de las dimensiones en los participantes del estudio se observa que la dimensión mayor es la de Auto-trascendencia (5.41), la segunda es Conservación (5.22), la tercera es Apertura al cambio (4.80) y en cuarto lugar Autopromoción (4.29). Para este grupo de participantes, lo relevante tiene que ver con el espíritu de servicio, justicia social, igualdad. Es la búsqueda del bien común. Lo anterior resulta congruente con el hecho que los participantes se forman para la docencia, carrera en la que esta dimensión adquiere un sentido necesario para el logro de los objetivos de formación.

Resulta interesante que el segundo puntaje fue para la dimensión de conservación, donde se aprecian la seguridad y el orden, en tercer lugar, fue para apertura al cambio, dimensión en la que se valora la independencia de acciones y pensamientos, lo que es congruente con la edad de la población, jóvenes de 17 a 30 años, cuya etapa evolutiva es precisamente en la que se define su sentido de diferenciación de los adultos y la búsqueda de sus propias ideas y desempeño y finalmente, autopromoción, donde se priorizan sus intereses propios.

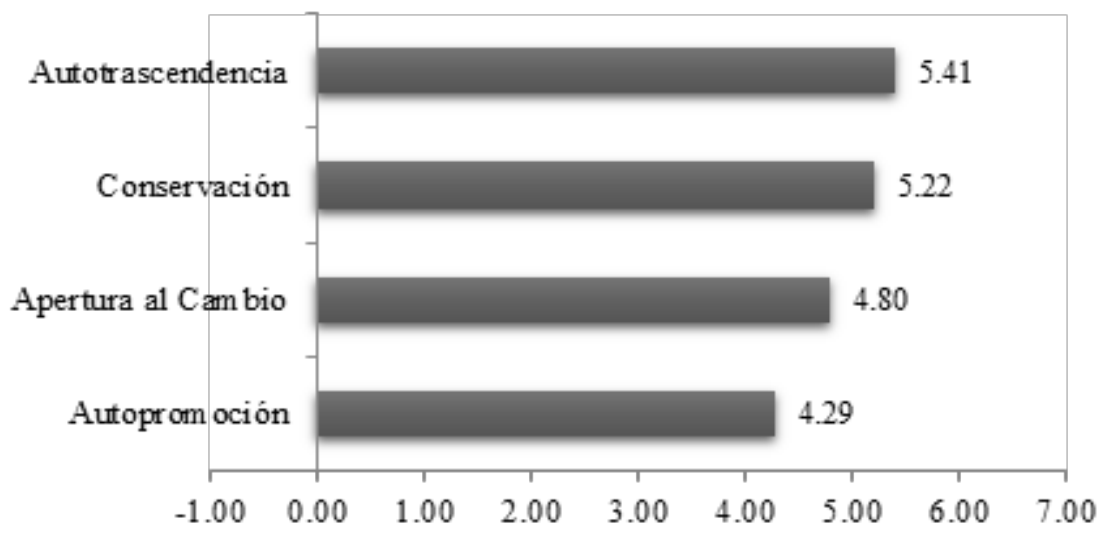

Figura 7. Dimensiones de valor en estudiantes de la UPNFM. Fuente: Elaboración Propia. Las dimensiones de mayor valor resultaron autotrascendencia y conservación. 
Factores asociados al perfil valórico de los estudiantes

El propósito de este análisis fue comparar los tipos de valor, valores individuales y valores colectivos a fin de establecer diferencias significativas entre los tipos de perfiles.

En relación a la variable de valores individuales, se obtuvo un promedio de 4.59 y de 5.33 en la variable valores colectivos, se observa la tendencia hacia los valores colectivos. Un análisis de significancia al interno de cada uno de estas variables revela que, para los valores individuales, hay una diferencia estadísticamente significativa $(\mathrm{t}=76.712 \mathrm{p}<0.05)$, ello es evidencia de que existe una gama diversa de puntuaciones dentro de este factor. Así, por ejemplo, el 29.3\% de los jóvenes universitarios, participantes en el estudio, tiene una puntuación menor a 4 puntos, lo que indica escasa presencia de valores individuales.

En este mismo sentido, el análisis de la variable valores colectivos, muestra también diferencias significativas $(\mathrm{t}=95.80 \mathrm{p}<0.05)$, encontrándose que un $30 \%$ de los jóvenes universitarios tiene puntuaciones de menos de 4 puntos, lo que sugiere que hay un segmento de la población estudiantil cuyos valores colectivos son reducidos, situación que contrasta con las puntuaciones alcanzados por el 70\% de los jóvenes, que superan los 5 puntos, que puede ser calificados como niveles altos de colectivismo.

En cuanto a la variable sexo y su relación con los valores individuales y colectivos, se obtuvo en los valores individuales, un promedio de 4.58 en las mujeres y un promedio de 4.61 en los varones, no se encontraron diferencias significativas ( $t-0.213 \mathrm{p}>0.05)$. En el caso de los valores colectivos, se obtuvo un promedio de 5.42 en las mujeres y 5.18 en los varones. Se presentan diferencias significativas $(\mathrm{t}-2.12 \mathrm{p}<0.05)$. Desde el punto de vista estadístico, hay diferencias entre varones y mujeres, presentando las mujeres mayor presencia de valores colectivos que los varones.

Acerca de la variable edad, para efectos de este estudio se organizó por rangos, de acuerdo al desarrollo evolutivo en esta etapa: a) de 17 a 19, estudiantes que inician su carrera 
universitaria, b) de 20 a 24, jóvenes que se encuentran a mitad de su carrera, con mayor independencia de sus hogares de origen y c) de 25 a 30, que de acuerdo a la teoría ya son jóvenes adultos completando su carrera universitaria. A partir de la prueba estadística ANOVA, no se encontraron diferencias significativas de la variable edad con los valores individuales $(\mathrm{f}=1.021 \mathrm{p}>0.05)$ y de igual manera, no se encontraron diferencias significativas en relación a los valores colectivos $(\mathrm{f}=012 \mathrm{p}>0.05)$.

Se llevó a cabo el procedimiento de escalonamiento que parte de las respuestas que dieron los participantes en la investigación; a partir de ellas, se calcula las distancias que hay entre las respuestas que dieron a cada una de los valores, de tal manera, que sistemáticamente las personas se acercan o se alejan de cierto tipo de valores.

De acuerdo a Guerrero y Ramírez (2012, p. 2), el escalamiento multidimensional (MultiDimensional Scaling- MDS):

[...] es una técnica de representación espacial que trata de visualizar sobre un mapa un conjunto de estímulos (firmas, productos, candidatos políticos, ideas u otros artículos) cuya posición relativa se desea analizar. El propósito del MDS es transformar los juicios de similitud o preferencia llevados a cabo por una serie de individuos sobre un conjunto de objetos o estímulos en distancias susceptibles de ser representadas en un espacio multidimensional. El MDS está basado en la comparación de objetos o de estímulos, de forma que si un individuo juzga a los objetos A y B como los más similares entonces las técnicas de MDS colocarán a los objetos A y B en el gráfico de forma que la distancia entre ellos sea más pequeña que la distancia entre cualquier otro par de objetos.

En ese sentido, se realizó esta técnica estadística con el propósito de establecer la distancia o cercanía que tenían los tipos de valor propuestos por Schwartz, en este grupo de estudiantes universitarios. La siguiente figura muestra esta ubicación de los tipos de valor. 


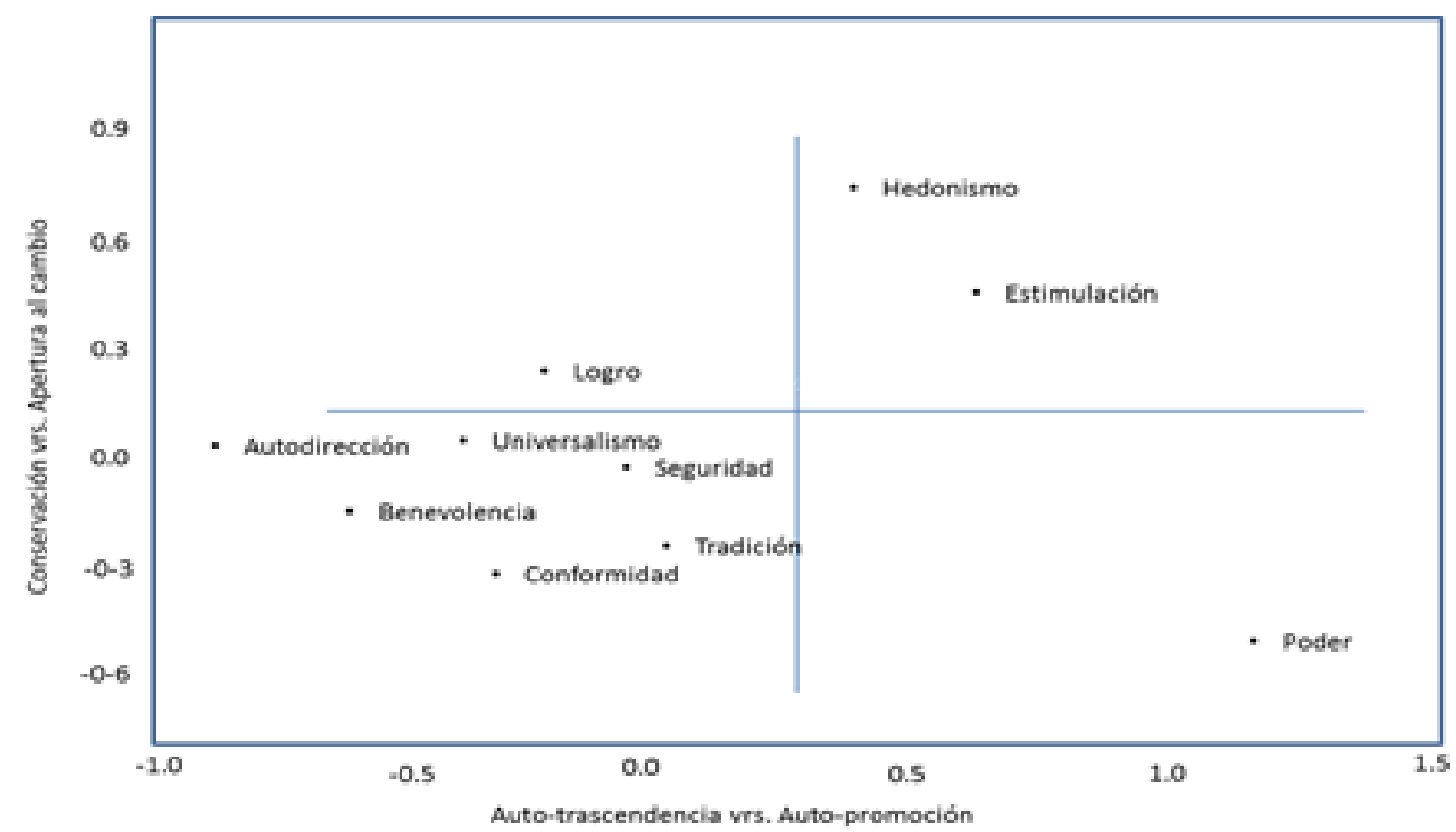

Figura 8. Escalonamiento de los valores en estudiantes de la UPNFM. Fuente: Elaboración propia con programa SPSS. Se aprecia en la figura que los tipos de valor se aproximan entre las dimensiones conservación y autotrascendencia, orientados a los valores colectivos.

La figura muestra dos contrastes, por una parte, los tipos de valor asociados a las dimensiones Conservación versus Apertura al Cambio y por otra, lo referido a las dimensiones Autotrascendencia versus Auto-promoción. Los resultados indican mayor proximidad en los tipos de valor universalismo, autodirección, benevolencia, seguridad, conformidad y tradición, cuyas metas se orientan a procurar el bienestar de los demás y en ese sentido declinar los intereses propios (sacrificio antes que el disfrute y lo social antes que lo individual).

Los tipos de valor hedonismo y estimulación se ubicaron con mayor distancia de los valores antes mencionados, lo cual es coincidente con la teoría que plantea que estos tipos de valor buscan el placer y el disfrute de lo novedoso a nivel individual. El tipo de valor poder se ubicó en una mayor distancia del resto de los demás valores y se orienta a lo individual. Finalmente, logro se visualiza cercano a los valores orientados a los valores de la dimensión conservación, pero se ubica un poco distante y más encaminado al disfrute de alcanzar las metas propuestas, en la dimensión apertura al cambio. 


\section{Conclusiones}

El acto de investigar en un campo del conocimiento específico, permite aproximarse a una profundización sistemática de un objeto de estudio, para dar cuenta de los hallazgos encontrados y establecer explicaciones razonadas que aporten al conocimiento.

Acercarse al estudio de los valores es algo complejo y en el caso particular de esta investigación, desarrollarlo con estudiantes universitarios es de mayor complejidad, puesto que se considera un grupo poblacional privilegiado por haber logrado acceder a la educación terciaria, en muchos casos serán los primeros en sus familias de origen de lograrlo, en otros casos, es el paso educativo obligado.

Valores de los estudiantes de la UPNFM: Una mirada desde la Teoría de Schwartz

El adoptar como sustento para esta investigación la perspectiva teórica del Dr. Shalom Schwartz, la cual ha sido revisada y puesta a prueba en numerosas oportunidades y contextos con resultados similares, permitió ser un referente conceptual importante sobre la temática, así como también ser un eje fundamental en la producción y el desarrollo de instrumentos de medición (véase Schwartz (1992), Schwartz y Blinsky (1987) y Delfino y Zubieta (2011).

Los resultados indican que, existe similitud en la estructura de valores que la teoría de valores que Schwartz propone sobre la configuración de los diez tipos de valor en esta población de estudiantes universitarios. Fue posible caracterizar los diez tipos de valor desde ambas aproximaciones metodológicas. A la vez, la posibilidad de identificar la importancia que los estudiantes otorgan a cada tipo de valor, como principio que guía su vida fue de aporte en el conocimiento de este constructo y la importancia que otorgan a los valores colectivos en relación con los valores individuales.

\section{Valores humanos: tensiones entre la persona y la comunidad}

La investigación realizada se acercó a la comprensión de los valores individuales y colectivos que poseen los estudiantes universitarios de la UPNFM. En esta aproximación se da cuenta de la inclinación que existe en esta población específica, por los valores colectivos, lo que tiene 
sentido dado que son personas que estudian para ser docentes y deberán hacerse cargo de los procesos de formación de otros seres humanos. Valores tales como conformidad, tradición, benevolencia, universalismo y seguridad, resultaron significativos para los estudiantes universitarios. Estos valores colectivos, promueven el cuidado e interés por otros y favorecen sin duda, los procesos de formación humana.

No obstante, este hecho, los valores individuales, aunque en menor porcentaje, tienen relativa importancia para los estudiantes universitarios. Por ejemplo, fue significativo el cuidado por sí mismos, el alcanzar sus metas de vida, buscar la novedad y la independencia, es así que, hedonismo, logro, autodirección, estimulación y poder, pudieron ser vistos como significativos en cierta ponderación.

Desde la universidad, es viable posibilitar un sano balance entre los valores colectivos que van a centrarse en la comunidad y los valores individuales que se enfocan en la persona, ambos necesarios en los procesos de formación humana y propiciar en el aula un espacio para dialogar sobre las tensiones que pudieran producirse en los estudiantes, cuando se inclinan por los unos o los otros.

\section{Referencias}

Arciniega, L., \& González, L. (2002). Valores individuales y valores coorporativos percibidos: Una aproximación empírica. Revista de Psicología Social Aplicada , 12 (1), 41-60.

Astill, B., Feather, N., \& Keeves, J. (2002). A multilevel analysis of the effects of parents, teachers and schools on student values. Social Psychology of Education, 5 (4), 345-363.

Bauman, Z. (2005). Los retos de la educación en la modernidad líquida. Barcelona: Editorial Gedisa.

Brinkmann, H., \& Bizama, M. (2000). Estructura psicológica de los valores: Presentación de una teoría. Sociedad Hoy, 4, 1-14.

Castro, A., \& Nader, M. (2006). La evaluación de los valores humanos con el Portrait Values Questionnarie de Schwartz. Interdisciplinaria, 23 (2), 155-174. 
Del Canto, E., \& Silva, A. (2013). Metodología cuantitativa: Abordaje desde la complementariedad en ciencias sociales. Revista de Ciencias Sociales, 3 (141), 25-34.

Delfino, G., \& Zubieta, E. (2011). Valores y política: análisis del perfil axiológico de los estudiantes universitarios de la ciudad de Buenos Aires (República de Argentina). Interdisciplinaria, Ciudad Autònoma de Buenos Aires, 28 (1), 93-114.

Fasce, E., Pérez, C., Ortiz, L., \& Parra, P. I. (2013). Aprendizaje autodirigido y su relación con el perfil valórico en estudiantes de medicina. Revista Medicina (141), 15-22.

Gallo, M. (2005). Masificación de la educaciòn superior: una reflexión acerca de sus causas y contradicciones. Facultad de Ciencias Económicas y Sociales UNMDP (22), 49- 63.

Guerrero, F., \& Ramírez, J. (2012). El análisis de escalamiento multidimensional: Una alternativa y un complemento a otras técnicas multivariantes. La sociología en sus escenarios (25), 1- 11.

Linares, L., Molpeceres, M., \& Musitu, G. (2001). La autoestima y las prioridades personales de valor: Un análisis de sus interrelaciones en la adolescencia. Anales de Psicología, 17 (2), 189-200.

Martínez, L., Silva, C., \& Hernández, A. (2010). ¿En qué ciudadanía creen los jóvenes? Creencias, aspiraciones de ciudadanía y motivaciones para la participación sociopolítica. Psykhé, 19 (2), 25-37.

Sampieri, R., Fernández-Collado, C., \& Baptistta, P. (2006). Metodología de la investigación (IV ed.). México: Mc Graw Hill.

Schwartz, S., \& Bilsky, W. (1987). Toward al psychological structure of human values. Journal of Personality and Social Psychology, 53 (3), 550-562.

Schwarz, S., \& Bilsky, W. (1990). Toward a theory of the universal content and structure of values: Extensions and cross-cultural replications. Journal of Personality and social Psychology, 58, 878-891. 
Schwartz, S. (1992). Universals in the content and structure of values: Theoretical advances and empirical tests in 20 countries. Advances in Experimiental Social Psychology, 25, 19-45.

Schwartz, S. (2006). Les valeurs de base de la personne: Théorie, measures et applications (Basic Human values: Theory, measurement and applications. Revue francaise de sociologie, 42, 249-288.

Suarez, M., \& Pérez, J. (2008). La disputa por la representación de los universitarios en México... o de cómo y para qué, forma la universidad pública a los jóvenes. En H. Suarez, \& J. Pérez, Jóvenes universitarios en Latinoamérica hoy (págs. 13-77). México: Miguel Angel Porrúa.

Tovar, J. (2007). Psicometría: tests psicométricos, confiabilidad y validez. Psicología: Tópicos de actualidad, 85-108.

Veliz, A., Dorner, A., Gonzáles, E., \& Ripoll, M. (2017). Perfil de valores de estudiantes de carreras de salud del sur de Chile. Horizonte Médico, 17 (2), 49-55. 